\title{
Horrocks Correspondence on a Quadric Surface
}

\author{
F. Malaspina* and A.P. Rao \\ Politecnico di Torino \\ Corso Duca degli Abruzzi 24, 10129 Torino, Italy \\ e-mail: malaspina@calvino.polito.it \\ University of Missouri-St. Louis \\ St Louis, MO, 63121, United States \\ e-mail: raoa@umsl.edu
}

June 24, 2018

\begin{abstract}
We extend the Horrocks correspondence between vector bundles and cohomology modules on the projective plane to the product of two projective lines. We introduce a set of invariants for a vector bundle on the product of two projective lines, which includes the first cohomology module of the bundle, and prove that there is a one to one correspondence between these sets of invariants and isomorphism classes of vector bundles without line bundle summands.
\end{abstract}

\section{Introduction}

We denote by $\mathbf{P}^{n}$ the $n$-dimensional projective space over a field $k$. For a vector bundle $E$ on $\mathbf{P}^{n}$, its intermediate cohomology modules are $H_{*}^{i}(E)=\bigoplus_{d \in \mathbb{Z}} H^{i}(E(d))(0<i<n)$, which are graded modules of finite length over the polynomial ring in $n+1$ variables. Horrocks in [26] established that a vector bundle on $\mathbf{P}^{n}$ is determined up to isomorphism and up to a sum of line bundles (i.e. up to stable equivalence) by its collection of intermediate cohomology modules and also a certain collection of extension classes involving these modules. In the simplest case, on $\mathbf{P}^{2}$, this Horrocks correspondence states that stable equivalence classes of vector bundles are in one-to-one correspondence with isomorphism classes of graded modules $M$ of finite length via the map $E \mapsto M=H_{*}^{1}(E)$. On $\mathbf{P}^{3}$, each triple $\left(M_{1}, M_{2}, \rho\right)$, where $M_{1}, M_{2}$ are graded modules of finite length and $\rho$ is an element in $\operatorname{Ext}^{2}\left(M_{1}^{\vee}, M_{2}^{\vee}\right)$, corresponds to the stable equivalence class of a vector bundle $E$ with $M_{1}, M_{2}$ as its intermediate cohomology modules.

This has led to attempts to study vector bundles on $\mathbf{P}^{n}$ with restrictions placed on the intermediate cohomology modules. Horrocks himself proved his splitting criterion (loc. cit.) which states that when all the intermediate cohomology modules are zero, the bundle is a

\footnotetext{
* Partially supported by GNSAGA of Indam (Italy)

Mathematics Subject Classification 2010: 14F05, 14J60.
}

Keywords: vector bundles, cohomology modules, smooth quadric surface 
sum of line bundles. In general, for an $n$-dimensional projective variety $(X, \mathcal{O}(1))$, a vector bundle $E$ on $X$ with all intermediate cohomology modules $H_{*}^{i}(E), 0<i<n$ equal to zero is called an ACM (Arithmetically Cohen-Macaulay) bundle. Thus, on $\mathbf{P}^{n}$, all ACM bundles are sums of line bundles of the form $\mathcal{O}(a)$. Refinements of this splitting criterion include the syzygy theorem for vector bundles on $\mathbf{P}^{n}([22,[23])$ and other splitting results for bundles with low ranks [30]. Several papers study the restrictions on cohomology modules of rank two bundles on $\mathbf{P}^{3}$ ([12, , 19, , 36]).

Horrocks' splitting criterion fails more generally. A subvariety $(X, \mathcal{O}(1))$ in projective space is called an arithmetically Cohen-Macaulay variety if the embedding is projectively normal and $\mathcal{O}$ itself is an ACM bundle. A smooth ACM subvariety $X$ which is not a linear subspace will always have ACM vector bundles that are not equal to sums of line bundles of the form $\mathcal{O}(a)$ (see [11] for smooth hypersurfaces in $\mathbf{P}^{n}$ and [33] Proposition 5 for an argument that works for any smooth ACM variety). On the $n$-dimensional quadric hypersurface $\mathcal{Q}_{n}$, the spinor bundles (see [34]) are such ACM bundles and Knörrer's theorem [27] states that all the ACM bundles on $\mathcal{Q}_{n}$ are direct sums of line bundles and twists of spinor bundles. ACM bundles on other varieties have been studied by many authors, in particular on hypersufaces ([11], 13], [14], [15], 16], [17], [18, [25], 31], 32], 37]), on complete intersections [9], on a few Fano threefolds ([1],[2],[10], 24]) and on Grassmannians of lines ([], [21]).

Ottaviani [35] has generalized the Horrocks splitting criterion to quadrics and to Grassmannians. In the last few years, extensions of the splitting criteria to other varieties have been made, as well as the cohomological characterization of certain types of vector bundles on these varieties $([4,[5],[6,[7,[20])$. These results represent special cases of what should be a Horrocks correspondence on other varieties. The aim of this paper is to obtain a Horrocks correspondence on a product of two projective lines. In other words, we find cohomological invariants that determine the isomorphism class of a vector bundle on the product of two projective lines.

Let $\mathcal{Q}$ be the smooth quadric in $\mathbf{P}^{3}$ defined by the equation $x_{0} x_{3}-x_{1} x_{2}$. Let $\{s, t\},\{u, v\}$ be coordinates on the factors of $\mathbf{P}^{1} \times \mathbf{P}^{1}$. Then $\mathcal{Q}$ is identified with $\mathbf{P}^{1} \times \mathbf{P}^{1}$, with $x_{0}, x_{1}, x_{2}, x_{3}$ corresponding to $s u, s v, t u, t v$. Let $\Sigma_{i}, i=1,2$, be the pull back of $\mathcal{O}_{\mathbf{P}^{1}}(1)$ under the two projections to $\mathbf{P}^{1}$. These are the spinor (line) bundles on $\mathcal{Q}$.

Let $E$ be a vector bundle on $\mathcal{Q}$ without any ACM summand, and let $M=M(E)$ denote $\bigoplus_{\nu \in \mathbb{Z}} H^{1}(\mathcal{Q}, E(\nu))$, which is a graded module of finite length over the homogeneous coordinate ring $S(\mathcal{Q})$. A free minimal presentation of $M$ as an $S(\mathcal{Q})$-module, when sheafified, yields an exact sequence of vector bundles on $\mathcal{Q}, 0 \rightarrow F_{M} \rightarrow L_{1} \rightarrow L_{0} \rightarrow 0$, where the $L_{i}$ are free and $H_{*}^{1}\left(F_{M}\right)=M$. Two auxiliary finite length $S(\mathcal{Q})$-modules $M_{\Sigma_{i}}=H_{*}^{1}\left(F_{M} \otimes \Sigma_{i}\right)$ can hence be defined. We will show that $E$ determines a graded vector subspace $W$ inside the $S(\mathcal{Q})$-module $M_{\Sigma_{1}}$, where this subspace is annihilated by multiplication by $u, v$. Such a subspace $W$ will be called a subspace of $\Sigma_{2}$-socle elements in $M_{\Sigma_{1}}$. Likewise, $E$ will determine a subspace $V$ in $M_{\Sigma_{2}}$ consisting of $\Sigma_{1}$-socle elements.

Given the collection of such data $\left\{M, W \subset M_{\Sigma_{1}}, V \subset M_{\Sigma_{2}}\right\}$, where $M$ is a graded $S(\mathcal{Q})$ module of finite length, and $W, V$ are as described above, an isomorphism $M \rightarrow M^{\prime}$ carries data $(M, W, V)$ to corresponding data $\left(M^{\prime}, W^{\prime}, V^{\prime}\right)$. Hence we can talk about isomorphism classes of this data. Rather than consider stable equivalence classes of vector bundles on $\mathcal{Q}$, we will consider isomorphism classes of vector bundles on $\mathcal{Q}$ without ACM summands.

We prove (3.2, 3.3)

Theorem. There is a bijection between isomorphism classes of vector bundles without ACM summands on $\mathcal{Q}$ and isomorphism classes of triples $(M, W, V)$, where $M$ is a graded $S(\mathcal{Q})$ - 
module of finite length, $W$ is a graded vector subspace of $\Sigma_{2}$-socle elements in $M_{\Sigma_{1}}, V$ is a graded vector subspace of $\Sigma_{1}$-socle elements in $M_{\Sigma_{2}}$.

We end the paper discussing examples that illustrate the invariants introduced. All extensions of Horrocks' ideas to other varieties require an understanding of ACM bundles on the variety. In cases when there are only finitely many irreducible ACM bundles (up to twist) on the variety, one can try to obtain a Horrocks correspondence as we have done here. In a paper in preparation, we consider the case of higher dimensional quadric hypersurfaces.

\section{The $\gamma$-sequence of a bundle}

Let $\mathcal{Q}$ be the smooth quadric surface in $\mathbf{P}^{3}$ as described above. The spinor (line) bundles $\Sigma_{1}, \Sigma_{2}$ on $\mathcal{Q}$ satisfy the canonical sequences (pulled back from $\mathbf{P}^{1}$ ):

$$
\begin{gathered}
0 \rightarrow \Sigma_{1}^{-1} \stackrel{[-t, s]^{\vee}}{\longrightarrow} 2 \mathcal{O} \stackrel{[s, t]}{\longrightarrow} \Sigma_{1} \rightarrow 0, \\
0 \rightarrow \Sigma_{2}^{-1} \stackrel{[-v, u]^{\vee}}{\longrightarrow} 2 \mathcal{O} \stackrel{[u, v]}{\longrightarrow} \Sigma_{2} \rightarrow 0 .
\end{gathered}
$$

$\Sigma_{1} \otimes \Sigma_{2}=\mathcal{O}(1)$ where $\mathcal{O}(1)$ denotes the restriction of the hyperplane bundle of $\mathbf{P}^{3}$ to $\mathcal{Q}$. We will sometimes denote $\Sigma_{1}$ by $\mathcal{O}(1,0), \Sigma_{2}$ by $\mathcal{O}(0,1)$ and $\mathcal{O}(1)$ by $\mathcal{O}(1,1)$, using the notation $\mathcal{O}(a, b)$ as well.

Let $S=k\left[x_{0}, x_{1}, x_{2}, x_{3}\right]$ be the polynomial ring and let $S(\mathcal{Q})$ be the coordinate ring of $\mathcal{Q}$. We will always view $S(\mathcal{Q})$ as a graded $k$-algebra with its grading inherited from $S$. For any sheaf $F$ on $\mathcal{Q}$, we define $H_{*}^{i}(F)$ to be the graded $S(\mathcal{Q})$-module $\bigoplus_{\nu \in \mathbb{Z}} H^{i}(\mathcal{Q}, F(\nu))$.

Definition 1.1. Let $F$ be a sheaf on $\mathcal{Q}$.

1. The graded $\Sigma_{1}$-socle sub-module of $H_{*}^{i}(F)$ is the kernel of the map

$$
\left.H_{*}^{i}(F) \stackrel{[-t, s]^{\vee}}{\longrightarrow} H_{*}^{i}\left(2 F \otimes \Sigma_{1}\right)\right)
$$

2. The graded $\Sigma_{2}$-socle sub-module of $H_{*}^{i}(F)$ is the kernel of the map

$$
\left.H_{*}^{i}(F) \stackrel{[-v, u]^{\vee}}{\longrightarrow} H_{*}^{i}\left(2 F \otimes \Sigma_{2}\right)\right)
$$

If $F$ is a vector bundle on $\mathcal{Q}, H_{*}^{1}(F)$ and its $\Sigma_{i}$-socle sub-modules are graded finite length $S(\mathcal{Q})$-modules. The $\Sigma_{i}$-socle sub-modules are annihilated by the forms $x_{0}, x_{1}, x_{2}, x_{3}$, hence their $S(\mathcal{Q})$-module structure descends to a graded $k$-vector space structure.

Lemma 1.2. Let $F$ be a sheaf on $\mathcal{Q}, V$ a finite-dimensional graded subspace consisting of $\Sigma_{i}$-socle elements in $H_{*}^{1}(F)$. Then there is a homomorphism $\alpha: V \otimes_{k} \Sigma_{i}^{-2} \rightarrow F$ such that $H_{*}^{1}(\alpha)$ has image $V$. For any other such map $\alpha^{\prime}$, there is an automorphism $\lambda$ of $V$ such that $H_{*}^{1}(\alpha \circ(\lambda \otimes 1))=H_{*}^{1}\left(\alpha^{\prime}\right)$.

Proof. Consider(for the case $\Sigma_{i}=\Sigma_{1}$ ) the canonical $\Sigma_{1}$ sequence (11) tensored by $F \otimes \Sigma_{1}$

$$
0 \rightarrow F \stackrel{[-t, s]^{\vee}}{\longrightarrow} 2 F \otimes \Sigma_{1} \stackrel{[s, t]}{\longrightarrow} F \otimes \Sigma_{1}^{2} \rightarrow 0 .
$$


There is a graded subspace $V^{\prime}$ of $H_{*}^{0}\left(F \otimes \Sigma_{1}^{2}\right)$ which is mapped isomorphically to $V \subset H_{*}^{1}(F)$. This induces a map $\alpha: V^{\prime} \otimes_{k} \mathcal{O} \rightarrow F \otimes \Sigma_{1}^{2}$ and thus the commuting diagram

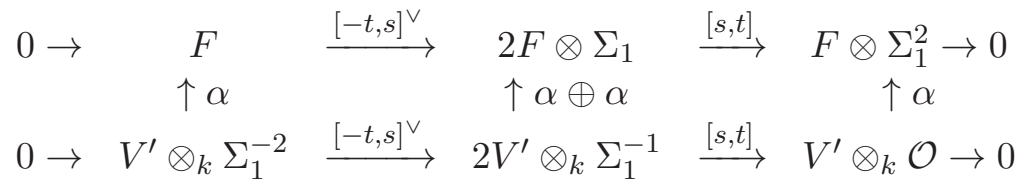

Then $H_{*}^{1}(\alpha): H_{*}^{1}\left(V^{\prime} \otimes_{k} \Sigma_{1}^{-2}\right) \rightarrow H_{*}^{1}(F)$ gives $V^{\prime} \cong V$.

Any other map $\alpha^{\prime}$ inducing a similar isomorphism must fit into a similar commutative diagram, with some $V^{\prime \prime} \subset H_{*}^{0}\left(F \otimes \Sigma_{1}^{2}\right)$. The map $H_{*}^{1}(\alpha)^{-1} \circ H_{*}^{1}\left(\alpha^{\prime}\right): V^{\prime \prime} \cong V \cong V^{\prime}=$ $H_{*}^{1}\left(V^{\prime} \otimes_{k} \Sigma_{1}^{-2}\right)$ is likewise induced by some $\lambda: V^{\prime \prime} \otimes_{k} \mathcal{O} \rightarrow V^{\prime} \otimes_{k} \mathcal{O}$ and it easy to verify that $\lambda$ is an isomorphism.

For any vector bundle $E$ on $\mathcal{Q}$, we define its first cohomology module $M(E)=H_{*}^{1}(E)$. For $\mathcal{O}, \Sigma_{i}, \Sigma_{i}^{-1}$, this module is zero, hence these three are ACM line bundles on $\mathcal{Q}$. Using the Künneth formula on $\mathbf{P}^{1} \times \mathbf{P}^{1}$, we can see that, as graded vector spaces, $M\left(\Sigma_{i}^{-2}\right)=k$, $M\left(\Sigma_{i}^{-3}\right)=k^{\oplus 2} \oplus k^{\oplus 2}$ (two components in degrees 0 and 1$)$.

Definition 1.3. A vector bundle $L$ on $\mathcal{Q}$ will be called free if $L \cong \bigoplus_{i=1}^{n} \mathcal{O}\left(a_{i}\right), a_{i} \in \mathbb{Z}$. $A$ vector bundle $E$ on $\mathcal{Q}$ has no $A C M$ summands if it cannot be expressed as $E^{\prime} \oplus L$ where $L$ is some line bundle of the form $\mathcal{O}(a), \Sigma_{1}(b), \Sigma_{2}(c)$.

Consider a finitely generated graded $S(\mathcal{Q})$-module $M$. It has a minimal free presentation as an $S(\mathcal{Q})$-module, given by a homogeneous map between free modules, where minimality means that the matrix of the map has no entry equal to a unit and no column equal to zero. This minimal presentation is unique up to isomorphism and any other free presentation of $M$ will split as the direct sum of the minimal presentation and a complex of free modules with the zero map.

In the special case when $M$ is a graded $S(\mathcal{Q})$-module of finite length, we may sheafify the minimal presentation to get a sequence of vector bundles on $\mathcal{Q}$ (since $M$ gives the zero sheaf on $\mathcal{Q})$ :

$$
\Psi: 0 \rightarrow F_{M} \rightarrow L_{1} \rightarrow L_{0} \rightarrow 0,
$$

where $L_{1}, L_{0}$ are free bundles, and $F_{M}$, unique up to isomorphism, is locally free on $\mathcal{Q}$, with $H_{*}^{1}\left(F_{M}\right)=M$. We will call $F_{M}$ the vector bundle associated to $M$.

Definition 1.4. Let $M$ be a graded $S(\mathcal{Q})$-module of finite length. Define $M_{\Sigma_{i}}$ to be the (finite length) graded $S(Q)$-module $H_{*}^{1}\left(F_{M} \otimes \Sigma_{i}\right)$, where $F_{M}$ is the vector bundle associated to $M$.

Let $E$ be a vector bundle on $\mathcal{Q}$. Since the invariant $M=H_{*}^{1}(E)$ is a graded $S(\mathcal{Q})$ module of finite length, we can attach to $E$ the invariants $M, F_{M}, M_{\Sigma_{1}}, M_{\Sigma_{2}}$. The short exact sequence obtained from the minimal presentation of $M$,

$$
\Psi: 0 \rightarrow F_{M} \rightarrow L_{1} \stackrel{\psi}{\rightarrow} L_{0} \rightarrow 0,
$$

gives an extension class $\Psi \in \operatorname{Ext}^{1}\left(L_{0}, F_{M}\right) \cong H^{1}\left(L_{0}^{\vee} \otimes F_{M}\right)$.

The construction of $\Psi$ started with a minimal presentation of $M$, hence a surjective homomorphism of $S(\mathcal{Q})$-modules $H_{*}^{0}\left(L_{0}\right) \rightarrow M$. Following Horrocks [26] (see also [8]), we have a second construction. From the homomorphism $H_{*}^{0}\left(L_{0}\right) \rightarrow M$, we get an element (of degree 0$)$ in $H_{*}^{0}\left(L_{0}\right)^{\vee} \otimes M \cong H_{*}^{1}\left(L_{0}^{\vee} \otimes E\right) \cong \operatorname{Ext}^{1}\left(L_{0}, E\right)$. Hence we get an exact sequence

$$
\gamma: 0 \rightarrow E \stackrel{f}{\rightarrow} A_{E} \stackrel{g}{\rightarrow} L_{0} \rightarrow 0,
$$


whose connecting homomorphism $H_{*}^{0}\left(L_{0}\right) \rightarrow H_{*}^{1}(E)$ returns the original map $H_{*}^{0}\left(L_{0}\right) \rightarrow M$. Therefore the bundle $A_{E}$ in the middle of $\gamma$ has $H_{*}^{1}\left(A_{E}\right)=0$. Thus $A_{E}$ is an ACM bundle $A_{E}$.

We call this sequence a $\gamma$-sequence for $E$, where $A_{E}$ is $\mathrm{ACM}$ and $L_{0}$ is free, with $H_{*}^{0}\left(L_{0}\right)$ minimally surjecting onto $H_{*}^{1}(E)$. There are natural actions of $\operatorname{Aut}\left(L_{0}\right)$ and $\operatorname{Aut}(E)$ on the collection of such extensions $\gamma$ for a fixed $E$.

Lemma 1.5. Let $E, E^{\prime}$ be bundles on $\mathcal{Q}$, with $\gamma$-sequences $\gamma: 0 \rightarrow E \stackrel{f}{\rightarrow} A_{E} \stackrel{g}{\rightarrow} L_{0} \rightarrow 0$, and $\gamma^{\prime}: 0 \rightarrow E^{\prime} \stackrel{f^{\prime}}{\rightarrow} A_{E^{\prime}} \stackrel{g^{\prime}}{\rightarrow} L_{0}^{\prime} \rightarrow 0$.

1. Any homomorphism $\sigma: E \rightarrow E^{\prime}$ gives an induced homomorphism $M(E) \rightarrow M\left(E^{\prime}\right)$ and an induced a map of exact sequences $\gamma \rightarrow \gamma^{\prime}$ along with well-defined commutative diagrams

$$
\begin{gathered}
H^{1}\left(E \otimes \Sigma_{i}\right) \stackrel{f}{\rightarrow} H_{*}^{1}\left(A_{E} \otimes \Sigma_{i}\right) \rightarrow 0 \\
\downarrow \\
\downarrow \\
H^{1}\left(E^{\prime} \otimes \Sigma_{i}\right) \stackrel{f^{\prime}}{\rightarrow} H_{*}^{1}\left(A_{E^{\prime}} \otimes \Sigma_{i}\right) \rightarrow 0
\end{gathered}
$$

for $i=1,2$.

2. If $\sigma$ induces an isomorphism $M(E) \rightarrow M\left(E^{\prime}\right)$, then $L_{0} \cong L_{0}^{\prime}$ and modulo the action of Aut $\left(L_{0}\right)$, we may take the map $\gamma \rightarrow \gamma^{\prime}$ of exact sequences to be the push-out of $\gamma$ by $\sigma$.

Proof. The first follows from the exact sequence

$$
0 \rightarrow \operatorname{Hom}\left(L_{0}, A_{E^{\prime}}\right) \stackrel{g}{\rightarrow} \operatorname{Hom}\left(A_{E}, A_{E^{\prime}}\right) \stackrel{f}{\rightarrow} \operatorname{Hom}\left(E, A_{E^{\prime}}\right) \rightarrow \operatorname{Ext}^{1}\left(L_{0}, A_{E^{\prime}}\right),
$$

where the last group is zero. In the resulting diagram

$$
\begin{aligned}
0 \rightarrow & E \stackrel{f}{\rightarrow} A_{E} \stackrel{g}{\rightarrow} L_{0} \rightarrow 0 \\
& \downarrow \sigma \quad \downarrow \sigma_{1} \quad \downarrow \sigma_{2} \\
0 & \rightarrow E^{\prime} \stackrel{f^{\prime}}{\rightarrow} A_{E^{\prime}} \stackrel{g^{\prime}}{\rightarrow} L_{0}^{\prime} \rightarrow 0
\end{aligned}
$$

the induced map $\sigma_{1}: A_{E} \rightarrow A_{E^{\prime}}$ is well-defined up to a map factoring through $L_{0}$, which would not change the map $\sigma_{1}: H_{*}^{1}\left(A_{E} \otimes \Sigma_{i}\right) \rightarrow H_{*}^{1}\left(A_{E^{\prime}} \otimes \Sigma_{i}\right)$.

For the second part, since both $L_{0}, L_{0}^{\prime}$ arise from minimal resolutions of the same module $M(E) \cong M\left(E^{\prime}\right)$, they are isomorphic, and furthermore $\sigma_{2}: H_{*}^{0}\left(L_{0}\right) \rightarrow H_{*}^{0}\left(L_{0}^{\prime}\right)$ is an isomorphism since it induces the isomorphism $\sigma: M(E) \rightarrow M\left(E^{\prime}\right)$.

Corollary 1.6. Let $E$ be a bundle on $\mathcal{Q}$ and let $M=M(E)$ and $F$ be the vector bundle associated to the module $M$. Then there is a homomorphism $\beta: F \rightarrow E$ such that $\gamma$ is the push-out by $\beta$ of $\Psi$ (modulo $\operatorname{Aut}\left(L_{0}\right)$ ).

Proof. Since both $H_{*}^{0}(\psi)$ and $H_{*}^{0}(g)$ present $M$ and since $H_{*}^{0}\left(L_{1}\right)$ is free, we can find a lifting $L_{1} \rightarrow A_{E}$ making the diagram commute. This gives a map $\beta: F \rightarrow E$ inducing an isomorphism on $H_{*}^{1}$.

The following proposition obtains conditions for comparing extensions $\gamma$ and $\gamma^{\prime}$ for two bundles $E$ and $E^{\prime}$. 
Proposition 1.7. Let $E, E^{\prime}$ be two vector bundles on $\mathcal{Q}$ with $M(E) \cong M\left(E^{\prime}\right) \cong M$. Let $F$ be the vector bundle associated to the module $M$, and let $\beta: F \rightarrow E, \beta^{\prime}: F \rightarrow E^{\prime}$ be morphisms for which $\gamma, \gamma^{\prime}$ are push-outs of $\Psi$. If $\operatorname{ker}\left[1 \otimes \beta: H_{*}^{1}\left(\Sigma_{i} \otimes F\right) \rightarrow H_{*}^{1}\left(\Sigma_{i} \otimes E\right)\right]$ equals $\operatorname{ker}\left[1 \otimes \beta^{\prime}: H_{*}^{1}\left(\Sigma_{i} \otimes F\right) \rightarrow H_{*}^{1}\left(\Sigma_{i} \otimes E^{\prime}\right)\right]$ for $i=1,2$, then there exists $\sigma: E \rightarrow E^{\prime}$ such that $\gamma^{\prime}$ is the push-out of $\gamma$ by $\sigma$, and $\sigma$ induces $M(E) \cong M\left(E^{\prime}\right)$.

Proof. The extension class $\gamma$ can be viewed (with abuse of notation) as the element in $H^{1}\left(L_{0}^{\vee} \otimes E\right)$ given by $\delta(\gamma)\left(I d_{L_{0}}\right)$ where $\delta(\gamma): H^{0}\left(L_{0}^{\vee} \otimes L_{0}\right) \rightarrow H^{1}\left(L_{0}^{\vee} \otimes E\right)$ is the connecting homomorphism, which is also (up to sign) the image of $I d_{E}$ in the connecting homomorphism $\delta\left(\gamma^{\vee}\right): H^{0}\left(E^{\vee} \otimes E\right) \rightarrow H^{1}\left(L_{0}^{\vee} \otimes E\right)$ for the dual short exact sequence $\gamma^{\vee}$.

Consider the commuting diagram derived from $\gamma^{\vee}$ :

$$
\begin{aligned}
& H^{0}\left(E^{\vee} \otimes E\right) \stackrel{\delta\left(\gamma^{\vee}\right)}{\longrightarrow} H^{1}\left(L_{0}^{\vee} \otimes E\right) \stackrel{g^{\vee} \otimes 1}{\longrightarrow} H^{1}\left(A_{E} \otimes E\right) \\
& \uparrow 1 \otimes \beta \quad \uparrow 1 \otimes \beta \\
& H^{1}\left(L_{0}^{\vee} \otimes F\right) \stackrel{g^{\vee} \otimes 1}{\longrightarrow} H^{1}\left(A_{E} \otimes F\right) .
\end{aligned}
$$

The element $\Psi \in H^{1}\left(L_{0}^{\vee} \otimes F\right)$ is pushed out by $\beta$ to the extension class $\gamma$ which is $\delta\left(\gamma^{\vee}\right)\left(I_{E}\right)$. Hence $(1 \otimes \beta) \circ\left(g^{\vee} \otimes 1\right)(\Psi)=\left(g^{\vee} \otimes 1\right) \circ(1 \otimes \beta)(\Psi)=0$. Since $A_{E}$ is ACM, $1 \otimes \beta: H^{1}\left(A_{E} \otimes F\right) \rightarrow H^{1}\left(A_{E} \otimes E\right)$ is a direct sum of maps $H^{1}\left(\Sigma_{i}(t) \otimes F\right) \rightarrow H^{1}\left(\Sigma_{i}(t) \otimes E\right)$ and $H^{1}(\mathcal{O}(t) \otimes F) \rightarrow H^{1}(\mathcal{O}(t) \otimes E), i=1,2, t \in \mathbb{Z}$. Since the last maps are isomorphisms, any non-zero components of $\left(g^{\vee} \otimes 1\right)(\Psi)$ in $H^{1}\left(A_{E} \otimes F\right)$ lie only in the summands of type $H^{1}\left(\Sigma_{i}(t) \otimes F\right)$ and they will be in the kernel of $1 \otimes \beta: H^{1}\left(\Sigma_{i}(t) \otimes F\right) \rightarrow H^{1}\left(\Sigma_{i}(t) \otimes E\right)$.

Consider the commuting diagram:

$$
\begin{aligned}
H^{0}\left(E^{\vee} \otimes E^{\prime}\right) \stackrel{\delta\left(\gamma^{\vee}\right)}{\longrightarrow} & H^{1}\left(L_{0}^{\vee} \otimes E^{\prime}\right) \stackrel{g^{\vee} \otimes 1}{\longrightarrow} H^{1}\left(A_{E} \otimes E^{\prime}\right) \\
\uparrow 1 \otimes \beta^{\prime} & \uparrow 1 \otimes \beta^{\prime} \\
& H^{1}\left(L_{0}^{\vee} \otimes F\right) \stackrel{g^{\vee} \otimes 1}{\longrightarrow} H^{1}\left(A_{E} \otimes F\right) .
\end{aligned}
$$

By corollary 1.6, $\left(1 \otimes \beta^{\prime}\right)(\Psi)$ represents $\gamma^{\prime}$, hence is non-zero. By our assumptions about the kernels of $1 \otimes \beta$ and $1 \otimes \beta^{\prime}$ on $H_{*}^{1}\left(\Sigma_{i} \otimes F\right),\left(1 \otimes \beta^{\prime}\right) \circ\left(g^{\vee} \otimes 1\right)(\Psi)=0$. Thus $\left(1 \otimes \beta^{\prime}\right)(\Psi)$ is the image of some element $\sigma \in H^{0}\left(E^{\vee} \otimes E^{\prime}\right)$, necessarily non-zero.

Consider the map $\sigma: E \rightarrow E^{\prime}$. Since $\sigma$ maps to $\gamma^{\prime}$ under $\delta\left(\gamma^{\vee}\right)$, it follows that the pushout of $\gamma$ under $\sigma$ is $\gamma^{\prime}$,

$$
\begin{aligned}
& 0 \rightarrow E \rightarrow A_{E} \rightarrow L_{0} \rightarrow 0 \\
& \downarrow \sigma \quad \downarrow \sigma_{1} \quad \| \\
& 0 \rightarrow E^{\prime} \rightarrow A_{E^{\prime}} \rightarrow L_{0} \rightarrow 0 .
\end{aligned}
$$

The induced map $H_{*}^{1}(E) \stackrel{\sigma}{\rightarrow} H_{*}^{1}\left(E^{\prime}\right)$ fits into the commuting diagram

$$
\begin{gathered}
H_{*}^{0}\left(L_{0}\right) \rightarrow H_{*}^{1}(E) \rightarrow 0 \\
\downarrow \sigma \\
\| \quad \rightarrow 0 . \\
H_{*}^{0}\left(L_{0}\right) \rightarrow H_{*}^{1}\left(E^{\prime}\right) \rightarrow 0
\end{gathered}
$$

Hence $H_{*}^{1}(E) \stackrel{\sigma}{\rightarrow} H_{*}^{1}\left(E^{\prime}\right)$ must be surjective, and since they are both isomorphic to the module $M$ which is a finite dimensional vector space over the base field, $H_{*}^{1}(E) \stackrel{\sigma}{\rightarrow} H_{*}^{1}\left(E^{\prime}\right)$ is an isomorphism. 


\section{The diagram of a bundle}

Let $E$ be a vector bundle on $\mathcal{Q}$. From the previous section, we get a graded $S(Q)$-module $M(E)$, a bundle $F$ associated to $M(E)$ and an ACM bundle $A_{E}$ with a push-out diagram

$$
\begin{aligned}
& \Psi: \quad 0 \rightarrow \rightarrow L_{1} \rightarrow L_{0} \rightarrow 0 \\
& \downarrow \beta \quad \downarrow \beta_{1} \quad \downarrow \beta_{2} \\
& \gamma: \quad 0 \rightarrow E \stackrel{f}{\rightarrow} A_{E} \stackrel{g}{\rightarrow} L_{0} \rightarrow 0 .
\end{aligned}
$$

Suppose $E=E^{\prime} \oplus L$ where $L$ is some line bundle of the form $\mathcal{O}(a), \Sigma_{1}(b), \Sigma_{2}(c)$. Then applying Lemma 1.5 to the projection $\sigma: E \rightarrow E^{\prime}$ we see that

$$
\gamma=\gamma^{\prime} \oplus\left\{0 \rightarrow L \stackrel{I d_{L}}{\longrightarrow} L \rightarrow 0 \rightarrow 0\right\}
$$

Hence we can "minimize $\gamma$ " by choosing $E$ to have no ACM summands.

By Knörrer's theorem [27, the ACM bundle $A_{E}$ has the form

$$
A_{E} \cong\left[\bigoplus_{i \in \mathbb{Z}} \mu_{i} \Sigma_{1}(i)\right] \oplus\left[\bigoplus_{j \in \mathbb{Z}} \nu_{j} \Sigma_{2}(j)\right] \oplus P_{E}=S_{E} \oplus P_{E}
$$

where $P_{E}$ is free.

We define the following numerical invariants associated to $E$ which determine $S_{E}$ :

$$
\mu=\left\{\mu_{i}\right\}_{i \in \mathbb{Z}} \text { and } \nu=\left\{\nu_{j}\right\}_{j \in \mathbb{Z}} .
$$

Furthermore, let $V$ be a graded vector space with dimensions $\mu, W$ with dimensions $\nu$. Then

$$
A_{E} \cong S_{E} \oplus P_{E} \cong\left(V \otimes_{k} \Sigma_{1}\right) \oplus\left(W \otimes_{k} \Sigma_{2}\right) \oplus P_{E} .
$$

Using the canonical spinor sequences (1), we get a canonical exact sequence $\Delta$ :

$$
\Delta: \quad 0 \rightarrow K_{E} \rightarrow L_{E} \oplus P_{E} \rightarrow A_{E} \rightarrow 0,
$$

where $K_{E}=\left(V \otimes \Sigma_{1}^{-1}\right) \oplus\left(W \otimes \Sigma_{2}^{-1}\right)$ and $L_{E}=(V \otimes 2 \mathcal{O}) \oplus(W \otimes 2 \mathcal{O})$.

Proposition 2.1. There is a commuting diagram

$$
\begin{aligned}
& \eta \quad \Delta \\
& 0 \quad 0 \\
& \downarrow \quad \downarrow \\
& K_{E} \quad=\quad K_{E} \\
& \downarrow \alpha \quad \downarrow \\
& 0 \rightarrow F \oplus Q_{E} \rightarrow L_{E} \oplus P_{E} \rightarrow L_{0} \quad \rightarrow 0 \\
& \downarrow \beta \quad \downarrow \quad \| \\
& \begin{array}{cccccccc}
\gamma: \quad 0 \rightarrow & E & \stackrel{f}{\rightarrow} & A_{E} & \stackrel{g}{\rightarrow} & L_{0} & \rightarrow 0 \\
& \downarrow & & \downarrow & & & \\
& 0 & & 0 & & &
\end{array}
\end{aligned}
$$

where $Q_{E}$ is free and the induced map $H_{*}^{1}(F) \rightarrow H_{*}^{1}(E)$ is an isomorphism. Furthermore, if $E$ has no ACM summands, then 
1. $H^{1}\left(\alpha \otimes I d_{\Sigma_{i}}\right): H_{*}^{1}\left(K_{E} \otimes \Sigma_{i}\right) \rightarrow H_{*}^{1}\left(F \otimes \Sigma_{i}\right)$ is injective for $i=1,2$.

2. the image of $H^{1}\left(\alpha \otimes I d_{\Sigma_{1}}\right)$ is a graded vector subspace of $H_{*}^{1}\left(F \otimes \Sigma_{1}\right)=M_{\Sigma_{1}}$ isomorphic to $W(1)$ and consists of $\Sigma_{2}$-socle elements.

3. the image of $H^{1}\left(\alpha \otimes I d_{\Sigma_{2}}\right)$ is a graded vector subspace of $H_{*}^{1}\left(F \otimes \Sigma_{2}\right)=M_{\Sigma_{2}}$ isomorphic to $V(1)$ and consists of $\Sigma_{1}$-socle elements.

Proof. Since $H_{*}^{0}\left(L_{E} \oplus P_{E}\right)$ is a free $S(\mathcal{Q})$-module and surjects onto $H_{*}^{0}\left(A_{E}\right)$, we obtain a composite map $H_{*}^{0}\left(L_{E} \oplus P_{E}\right) \rightarrow H_{*}^{0}\left(L_{0}\right)$ that gives a free presentation for $M(E)$ and hence can be compared with the minimal presentation $\Psi$ for $M(E)$. This gives the second row of the diagram where $Q_{E}$ is some free bundle. Since the bottom row is a push-out of the second row, $H_{*}^{1}(F) \rightarrow H_{*}^{1}(E)$ is an isomorphism. The sequence labelled $\eta$ is the pull-back by $f$ of the sequence $\Delta$.

Now suppose $E$ has no ACM summands. We wish to show that $H_{*}^{0}\left(E \otimes \Sigma_{i}\right) \rightarrow H_{*}^{1}\left(K_{E} \otimes \Sigma_{i}\right)$ is the zero map.

Indeed, suppose that when $i=1$, a section $s$ of $E \otimes \Sigma_{1}(p)$ maps to a nonzero element of $H^{1}\left(K_{E} \otimes \Sigma_{1}(p)\right)$. Then the image $s^{\prime}$ of $s$ in $H^{0}\left(A_{E} \otimes \Sigma_{1}(p)\right)$ maps to a nonzero element of $H^{1}\left(K_{E} \otimes \Sigma_{1}(p)\right)$. But the second column is built out of a direct sum of the canonical sequences (11) (twisted by integers $q$ ). Tensoring such a sequence with $\Sigma_{1}(p)$ gives

$$
0 \rightarrow \Sigma_{j}^{-1}(q) \otimes \Sigma_{1}(p) \rightarrow 2 \mathcal{O}(q) \otimes \Sigma_{1}(p) \rightarrow \Sigma_{j}(q) \otimes \Sigma_{1}(p) \rightarrow 0 .
$$

The induced map from $H^{0}$ to $H^{1}$ is nonzero only if $j=2, p+q=-1$, and in this case, the element 1 in $H^{0}\left(\Sigma_{2} \otimes \Sigma_{1}(-1)\right)=H^{0}(\mathcal{O})$ maps to a nonzero element.

Hence there is a summand of $A_{E} \otimes \Sigma_{1}(p)$ of the form $\mathcal{O}$, such that $s^{\prime}$ projects to the global section 1 of this summand. Thus $E \otimes \Sigma_{1}(p)$ has $\mathcal{O}$ as a summand, giving $\Sigma_{2}(p-1)$ as an ACM summand of $E$.

The last statements follow from the the fact that (for $\Sigma_{1}$ )

$$
H_{*}^{1}\left(K_{E} \otimes \Sigma_{1}\right)=H_{*}^{1}\left(W \otimes_{k} \Sigma_{2}^{-1} \otimes \Sigma_{1}\right)=H_{*}^{1}\left(W(1) \otimes_{k} \mathcal{O}(0,-2)\right)=W(1)
$$

and from the commutative diagram

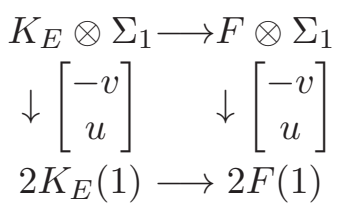

where $H_{*}^{1}\left(K_{E}\right)=0$.

Definition 2.2. The diagram in Proposition [2.1] will be called a diagram of E.

Proposition 2.3. For $i=1,2$, there is an exact sequence

$$
0 \rightarrow H_{*}^{1}\left(K_{E} \otimes \Sigma_{i}\right) \stackrel{\alpha}{\rightarrow} H_{*}^{1}\left(F \otimes \Sigma_{i}\right) \stackrel{\beta}{\rightarrow} H_{*}^{1}\left(E \otimes \Sigma_{i}\right) \stackrel{f}{\rightarrow} H_{*}^{1}\left(A_{E} \otimes \Sigma_{i}\right) \rightarrow 0
$$

obtained from the diagram of $E$. 
Proof. It suffices to verify exactness at $H_{*}^{1}\left(E \otimes \Sigma_{1}\right)$. The diagram

$$
\begin{array}{cc}
2 H_{*}^{0}\left(L_{0}\right) & \rightarrow 2 H_{*}^{1}(E) \rightarrow 0 \\
\downarrow & \rightarrow s, t \quad \downarrow s, t \\
H_{*}^{0}\left(L_{0} \otimes \Sigma_{1}\right) \rightarrow H_{*}^{1}\left(E \otimes \Sigma_{1}\right) \stackrel{f}{\rightarrow} H_{*}^{1}\left(A_{E} \otimes \Sigma_{1}\right) \rightarrow 0 \\
\downarrow \\
0
\end{array}
$$

shows that ker $f$ equals the image of $2 H_{*}^{1}(E) \stackrel{s, t}{\longrightarrow} H_{*}^{1}\left(E \otimes \Sigma_{1}\right)$. As a special case, when $E$ is $F$, it follows that $2 H_{*}^{1}(F) \stackrel{s, t}{\longrightarrow} H_{*}^{1}\left(F \otimes \Sigma_{1}\right)$ is onto. Now the diagram

$$
\begin{array}{ccc}
2 H_{*}^{1}(F) & \stackrel{\cong}{\rightrightarrows} & 2 H_{*}^{1}(E) \\
\downarrow s, t & \downarrow s, t \\
H^{1}\left(F \otimes \Sigma_{1}\right) \stackrel{\beta}{\rightarrow} H_{*}^{1}\left(E \otimes \Sigma_{1}\right) \\
\downarrow \\
0
\end{array}
$$

shows that $\operatorname{ker} f$ is the image of $\beta$.

The following theorem compares extensions $\eta: 0 \rightarrow K_{E} \stackrel{\alpha}{\rightarrow} F \oplus Q_{E} \stackrel{\beta}{\rightarrow} E \rightarrow 0$ and $\eta^{\prime}: 0 \rightarrow K_{E^{\prime}} \stackrel{\alpha^{\prime}}{\rightarrow} F^{\prime} \oplus Q_{E^{\prime}} \stackrel{\beta^{\prime}}{\rightarrow} E^{\prime} \rightarrow 0$ for two bundles $E$ and $E^{\prime}$.

Theorem 2.4. Let $E, E^{\prime}$ be two vector bundles on $\mathcal{Q}$ with no $A C M$ summands. Suppose $M(E)=M\left(E^{\prime}\right), K_{E}=K_{E^{\prime}}$. In the diagram of proposition [2.1, suppose for $i=1,2$, $H^{1}\left(\alpha \otimes I d_{\Sigma_{i}}\right)$ and $H^{1}\left(\alpha^{\prime} \otimes I d_{\Sigma_{i}}\right)$ have the same image in $H_{*}^{1}\left(F \otimes \Sigma_{i}\right)$. Then there is a homomorphism $\sigma: E \rightarrow E^{\prime}$ such that the extension class $\eta$ is the pull-back by $\sigma$ of the extension $\eta^{\prime}$ (up to an automorphism of $K_{E}=K_{E^{\prime}}$ ).

Proof. With $K_{E}=K_{E^{\prime}}=K$, we have the two commuting diagrams

$$
\begin{aligned}
& H^{0}\left(E^{\vee} \otimes E\right) \stackrel{\delta(\eta)}{\longrightarrow} H^{1}\left(E^{\vee} \otimes K\right) \stackrel{1 \otimes \alpha}{\longrightarrow} H^{1}\left(E^{\vee} \otimes\left(F \oplus Q_{E}\right)\right) \\
& \uparrow(f)^{\vee} \otimes 1 \quad \uparrow(f)^{\vee} \otimes 1 \\
& H^{1}\left(A_{E}^{\vee} \otimes K\right) \stackrel{1 \otimes \alpha}{\longrightarrow} H^{1}\left(A_{E}^{\vee} \otimes\left(F \oplus Q_{E}\right)\right)
\end{aligned}
$$

and

$$
\begin{aligned}
& H^{0}\left(E^{\vee} \otimes E^{\prime}\right) \stackrel{\delta\left(\eta^{\prime}\right)}{\longrightarrow} H^{1}\left(E^{\vee} \otimes K\right) \stackrel{1 \otimes \alpha^{\prime}}{\longrightarrow} H^{1}\left(E^{\vee} \otimes\left(F \oplus Q_{E^{\prime}}\right)\right) \\
& \uparrow(f)^{\vee} \otimes 1 \quad \uparrow(f)^{\vee} \otimes 1 \\
& H^{1}\left(A_{E}^{\vee} \otimes K\right) \stackrel{1 \otimes \alpha^{\prime}}{\longrightarrow} H^{1}\left(A_{E}^{\vee} \otimes\left(F \oplus Q_{E^{\prime}}\right)\right) .
\end{aligned}
$$

Let $K=V \otimes \Sigma_{1}^{-1} \oplus W \otimes \Sigma_{2}^{-1}$ for graded vector spaces $V, W$. By the assumption on the images of $H_{*}^{1}\left(K \otimes \Sigma_{i}\right)$ in $H_{*}^{1}\left(F \otimes \Sigma_{i}\right)$ and using lemma 1.2, we can assume there are automorphisms $\lambda_{1}, \lambda_{2}$ of $V, W$ such that if $\lambda=\left[\begin{array}{cc}\lambda_{1} & 0 \\ 0 & \lambda_{2}\end{array}\right]: K \rightarrow K$, then the maps $H^{1}\left(1 \otimes \alpha^{\prime}\right)$ 
and $H^{1}(1 \otimes(\alpha \circ \lambda))$ from $H_{*}^{1}\left(\Sigma_{i}^{-1} \otimes K\right)$ to $H_{*}^{1}\left(\Sigma_{i}^{-1} \otimes\left(F \oplus Q_{E^{\prime}}\right)\right)$ are equal. (note that $H_{*}^{1}\left(\Sigma_{i}^{-1} \otimes Q_{E^{\prime}}\right)=0$.) Since $A_{E}^{\vee}$ is a direct sum of terms of the form $\mathcal{O}(t), \Sigma_{i}^{-1}(t)$, it follows that the maps $H^{1}\left(1 \otimes \alpha^{\prime}\right)$ and $H^{1}(1 \otimes(\alpha \circ \lambda))$ from $H_{*}^{1}\left(A_{E}^{\vee} \otimes K\right)$ to $H_{*}^{1}\left(A_{E}^{\vee} \otimes\left(F \oplus Q_{E^{\prime}}\right)\right)$ are equal.

In the first diagram, the element $I d_{E} \in H^{0}\left(E^{\vee} \otimes E\right)$ maps to $\eta \in H^{1}\left(E^{\vee} \otimes K\right)$. Hence $(1 \otimes \alpha) \circ\left((f)^{\vee} \otimes 1\right)(\Delta)=0$. Thus also $\left((f)^{\vee} \otimes 1\right) \circ(1 \otimes \alpha)(\Delta)=0$.

Hence $\left((f)^{\vee} \otimes 1\right) \circ\left(1 \otimes \alpha^{\prime}\right)(1 \otimes \lambda)^{-1}(\Delta)=0$ and thus $\left.\left(1 \otimes \alpha^{\prime}\right) \circ(f)^{\vee} \otimes 1\right)\left((1 \otimes \lambda)^{-1}(\Delta)=0\right.$. $(1 \otimes \lambda)^{-1}(\Delta)$ is non-zero, and $(f)^{\vee} \otimes 1$ is injective on $H^{1}\left(A_{E}^{\vee} \otimes K\right)$, hence there is a non-zero $\sigma \in H^{0}\left(E^{\vee} \otimes E^{\prime}\right)$ with the property that the pull-back by $\sigma$ of the sequence $\eta^{\prime}$ is $(1 \otimes \lambda)^{-1}(\eta)$. Thus

$$
\begin{array}{r}
0 \rightarrow K \stackrel{\alpha^{\prime}}{\rightarrow} F \oplus Q_{E^{\prime}} \stackrel{\beta^{\prime}}{\rightarrow} E^{\prime} \rightarrow 0 \\
\uparrow \lambda \quad \uparrow \tilde{\sigma} \quad \uparrow \sigma \\
0 \rightarrow K \stackrel{\alpha}{\rightarrow} F \oplus Q_{E} \stackrel{\beta}{\rightarrow} E \rightarrow 0 .
\end{array}
$$

\section{Invariants of a bundle}

We have seen that a vector bundle $E$ on $\mathcal{Q}$ with no ACM summands determines the following invariants.

- A graded $S(\mathcal{Q})$-module $M=H_{*}^{1}(E)$ of finite length, hence an associated bundle $F$ and associated modules $M_{\Sigma_{1}}, M_{\Sigma_{2}}$.

- Finitely supported sequences $\mu=\left\{\mu_{i}\right\}_{i \in \mathbb{Z}}, \nu=\left\{\nu_{j}\right\}_{j \in \mathbb{Z}}$ of positive integers (determined by $\left.A_{E}\right)$. Hence graded vector spaces $V, W$ with graded dimensions determined by $\mu, \nu$.

- In the module $M_{\Sigma_{1}}$, a graded subspace of $\Sigma_{2}$-socle elements isomorphic to $W(1)$.

- In the module $M_{\Sigma_{2}}$, a graded subspace of $\Sigma_{1}$-socle elements isomorphic to $V(1)$.

Any bundle in the stable equivalence class of $E$ determines the same data as $E$. Replacing $E$ by $E(e)$ changes this data by a corresponding shift in grading.

Lemma 3.1. Given an isomorphism $\phi: M \rightarrow M^{\prime}$, of graded $S(\mathcal{Q})$-modules of finite length There are well-defined induced isomorphisms $M_{\Sigma_{i}} \cong M_{\Sigma_{i}}^{\prime}$.

Proof. Indeed, given the map $\phi: M \rightarrow M^{\prime}$, there is an induced map from the sequence $\Psi: 0 \rightarrow F \rightarrow L_{1} \rightarrow L_{0} \rightarrow 0$ to the corresponding sequence $\Psi^{\prime}: 0 \rightarrow F^{\prime} \rightarrow L_{1}^{\prime} \rightarrow L_{0}^{\prime} \rightarrow 0$. The map $F \rightarrow F^{\prime}$ is an isomorphism, unique up to a term that factors through $L_{1}$. Hence the induced map $H_{*}^{1}\left(F \otimes \Sigma_{i}\right) \rightarrow H_{*}^{1}\left(F^{\prime} \otimes \Sigma_{i}\right)$ is well-determined by $\phi$.

Theorem 3.2. (Uniqueness) Given $E, E^{\prime}$ two bundles on $\mathcal{Q}$ without $A C M$ summands, with invariants $M, W(1) \subset M_{\Sigma_{1}}, V(1) \subset M_{\Sigma_{2}}$ and $M^{\prime}, W^{\prime}(1) \subset M_{\Sigma_{1}}^{\prime}, V^{\prime}(1) \subset M_{\Sigma_{2}}^{\prime}$. Suppose $\exists \phi: M \cong M^{\prime}$, such that the induced isomorphisms $M_{\Sigma_{i}} \cong M_{\Sigma_{i}}^{\prime}$ carry $V(1)$ to $V^{\prime}(1)$ and $W(1)$ to $W^{\prime}(1)$. Then $E$ and $E^{\prime}$ are isomorphic.

Proof. We may assume that $M=M^{\prime}$ and that there is an automorphism $\phi$ of $F$ which carries $V(1)$ to $V^{\prime}(1)$ and $W(1)$ to $W^{\prime}(1)$. Since the graded vector spaces $V, W$ are isomorphic to $V^{\prime}, W^{\prime}$, we may assume that $K_{E}=K_{E^{\prime}}=K$. In the diagram of proposition 2.1 for $E^{\prime}$, we may replace $\alpha^{\prime}$ by $\phi^{-1} \circ \alpha^{\prime}$ etc. and assume that $\alpha$ and $\alpha^{\prime}$ give the same images in 
$H_{*}^{1}\left(F \otimes \Sigma_{i}\right)$. By applying Theorem 2.4, there exists $\sigma: E \rightarrow E^{\prime}$ and $\lambda: K \cong K$ such that there is a commutative diagram

$$
\begin{gathered}
0 \rightarrow K \stackrel{\alpha^{\prime}}{\rightarrow} F \oplus Q_{E^{\prime}} \stackrel{\beta^{\prime}}{\rightarrow} E^{\prime} \rightarrow 0 \\
\uparrow \lambda \quad \uparrow \tilde{\sigma} \quad \uparrow \sigma \\
0 \rightarrow K \stackrel{\alpha}{\rightarrow} F \oplus Q_{E} \stackrel{\beta}{\rightarrow} E \rightarrow 0 .
\end{gathered}
$$

This gives a diagram of cohomology

$$
\begin{aligned}
& 0 \rightarrow H_{*}^{1}\left(K \otimes \Sigma_{i}\right) \stackrel{\alpha^{\prime}}{\rightarrow} H_{*}^{1}\left(F \otimes \Sigma_{i}\right) \stackrel{\beta^{\prime}}{\rightarrow} H_{*}^{1}\left(E^{\prime} \otimes \Sigma_{i}\right) \rightarrow H_{*}^{2}\left(K \otimes \Sigma_{i}\right) \\
& \begin{array}{llll}
\tau \lambda & \uparrow & \uparrow \sigma & \uparrow \lambda
\end{array} \\
& 0 \rightarrow H_{*}^{1}\left(K \otimes \Sigma_{i}\right) \stackrel{\alpha}{\rightarrow} H_{*}^{1}\left(F \otimes \Sigma_{i}\right) \stackrel{\beta}{\rightarrow} H_{*}^{1}\left(E \otimes \Sigma_{i}\right) \rightarrow H_{*}^{2}\left(K \otimes \Sigma_{i}\right)
\end{aligned}
$$

where since $\lambda: H_{*}^{2}\left(K \otimes \Sigma_{i}\right) \rightarrow H_{*}^{2}\left(K \otimes \Sigma_{i}\right)$ is an isomorphism, we get coker $\beta \hookrightarrow$ coker $\beta^{\prime}$.

By Lemma 1.5, $\sigma$ also induces

$$
\begin{aligned}
\gamma^{\prime}: & 0 \rightarrow E^{\prime} \stackrel{f^{\prime}}{\rightarrow} A_{E^{\prime}} \stackrel{g^{\prime}}{\rightarrow} L_{0} \rightarrow 0 \\
& \uparrow \sigma \quad \uparrow \sigma_{1} \stackrel{\uparrow \sigma_{2}}{ } \\
\gamma^{\prime}: \quad & 0 \rightarrow E \stackrel{f}{\rightarrow} A_{E} \stackrel{g}{\rightarrow} L_{0} \rightarrow 0
\end{aligned}
$$

By Lemma 2.3, we get

$$
\begin{gathered}
0 \rightarrow H_{*}^{1}\left(K \otimes \Sigma_{i}\right) \stackrel{\alpha^{\prime}}{\rightarrow} H_{*}^{1}\left(F \otimes \Sigma_{i}\right) \stackrel{\beta^{\prime}}{\rightarrow} H_{*}^{1}\left(E^{\prime} \otimes \Sigma_{i}\right) \rightarrow H_{*}^{1}\left(A_{E^{\prime}} \otimes \Sigma_{i}\right) \rightarrow 0 \\
\uparrow \lambda \quad \uparrow \tilde{\sigma} \\
0 \rightarrow H_{*}^{1}\left(K \otimes \Sigma_{i}\right) \stackrel{\alpha}{\rightarrow} H_{*}^{1}\left(F \otimes \Sigma_{i}\right) \stackrel{\beta}{\rightarrow} H_{*}^{1}\left(E \otimes \Sigma_{i}\right) \rightarrow H_{*}^{1}\left(A_{E} \otimes \Sigma_{i}\right) \rightarrow 0
\end{gathered}
$$

Hence, coker $\beta$ and coker $\beta^{\prime}$ can be identified with $H_{*}^{1}\left(A_{E} \otimes \Sigma_{i}\right)=H_{*}^{1}\left(A_{E^{\prime}} \otimes \Sigma_{i}\right)$. Therefore, $\sigma_{1}: H_{*}^{1}\left(A_{E} \otimes \Sigma_{i}\right) \rightarrow H_{*}^{1}\left(A_{E^{\prime}} \otimes \Sigma_{i}\right)$ is an inclusion of vector spaces of the same rank, hence is an isomorphism.

Thus there exists $\sigma: E \rightarrow E^{\prime}$ such that the induced map $\sigma_{1}: H_{*}^{1}\left(A_{E} \otimes \Sigma_{i}\right) \rightarrow H_{*}^{1}\left(A_{E^{\prime}} \otimes \Sigma_{i}\right)$ described in Lemma 1.5] is an isomorphism for each $i$. Again, by Theorem 1.7, there exists $\sigma: E \rightarrow E^{\prime}$ such that $\sigma$ induces $M(E) \cong M\left(E^{\prime}\right)$ (and $\gamma^{\prime}$ is the push-out of $\gamma$ by $\sigma$ ). By the Zariski openness of these conditions, the general $\sigma: E \rightarrow E^{\prime}$ has the property that in the induced diagram

$$
\begin{aligned}
& \gamma^{\prime}: 0 \rightarrow E^{\prime} \stackrel{f^{\prime}}{\rightarrow} A_{E^{\prime}} \stackrel{g^{\prime}}{\rightarrow} L_{0} \rightarrow 0 \\
& \uparrow \sigma \quad \uparrow \sigma_{1} \quad \uparrow \sigma_{2} \\
& \gamma^{\prime}: \quad 0 \rightarrow E \stackrel{f}{\rightarrow} A_{E} \stackrel{g}{\rightarrow} L_{0} \rightarrow 0,
\end{aligned}
$$

$\sigma_{2}$ is an isomorphism, and $\sigma_{1}: H_{*}^{1}\left(A_{E} \otimes \Sigma_{i}\right) \cong H_{*}^{1}\left(A_{E^{\prime}} \otimes \Sigma_{i}\right)$ for $i=1,2$.

The map $\sigma_{1}: A_{E}=S \oplus P_{E} \rightarrow A_{E^{\prime}}=S \oplus P_{E^{\prime}}$ can be written as $\left[\begin{array}{ll}\sigma_{11} & \sigma_{12} \\ \sigma_{21} & \sigma_{22}\end{array}\right]$. Then $\sigma_{11}$ has the property that $H^{1}\left(\sigma_{11}\right): H_{*}^{1}\left(S \otimes \Sigma_{i}\right) \rightarrow H_{*}^{1}\left(S \otimes \Sigma_{i}\right)$ is an isomorphism. Lemma 1.2 shows that $\sigma_{11}$ is an isomorphism from $S \rightarrow S$. 
Now we can apply automorphisms to $A_{E}, A_{E^{\prime}}$ and assume that $\sigma_{1}=\left[\begin{array}{cc}1_{S} & 0 \\ 0 & \sigma_{22}\end{array}\right]$. The bundle $E^{\prime}$ has no free summands. Hence any section $s$ of $E^{\prime}$ maps to $(a, b) \in S \oplus P_{E^{\prime}}$ where $b$ is a column of polynomials where no entry in the column is a non-zero scalar. Let $e$ be a minimal generator for $H_{*}^{0}\left(P_{E^{\prime}}\right)$ (for example $[1,0, \ldots, 0]^{\vee}$ ). From the commuting diagram of long exact sequences, we see that there is a section $s$ of $E^{\prime}$ such that $(-a, e-b)$ is in the image of $\sigma_{1}$. Hence $e-b$ is in the image of $\sigma_{22}$, which says that $\sigma_{22}$ is surjective on global sections.

In particular, now $\sigma: E \rightarrow E^{\prime}$ is also surjective as a map of sheaves. Thus $E$ has rank not less than that of $E^{\prime}$. The discussion can be reversed to show that $E, E^{\prime}$ have the same rank, and so $\sigma: E \rightarrow E^{\prime}$ is really an isomorphism.

We finally ask the question: For given data of invariants, is there a vector bundle $E$ on $\mathcal{Q}$ with those invariants?

Theorem 3.3. (Existence) Let $M$ be a graded $S(\mathcal{Q})$-module of finite length. Let $\mu$ and $\nu$ be two sequences of positive integers with finite support, $V, W$ the corresponding graded vector spaces. Assume that $M_{\Sigma_{1}}$ has a graded vector subspace isomorphic to $W(1)$ consisting of $\Sigma_{2}$-socle elements and $M_{\Sigma_{2}}$ has a graded vector subspace isomorphic to $V(1)$ of $\Sigma_{1}$-socle elements. Then there exists a bundle $E$ with no ACM summands on $\mathcal{Q}$ with these invariants.

Proof. Let $F$ be the bundle associated to $M$, with $\Psi: 0 \rightarrow F \rightarrow L_{1} \stackrel{\phi}{\rightarrow} L_{0} \rightarrow 0$ given by a minimal presentation of $M$. Consider the sequence

$$
0 \rightarrow F \otimes \Sigma_{1}(-1) \stackrel{[-v, u]^{\vee}}{\longrightarrow} 2 F \rightarrow F \otimes \Sigma_{2} \rightarrow 0
$$

In the cohomology sequence $H_{*}^{0}\left(F \otimes \Sigma_{2}\right) \rightarrow H_{*}^{1}\left(F \otimes \Sigma_{1}(-1)\right) \stackrel{[-v, u]^{\vee}}{\longrightarrow} H_{*}^{1}(2 F)$ obtained from this, the graded vector subspace $W$ is annihilated by $[-v, u]^{\vee}$, hence there is a subspace in $H_{*}^{0}\left(F \otimes \Sigma_{2}\right)$ (not necessarily unique) that maps isomorphically to $W$. By abuse of notation, we call this also as $W$. We get a map $W \otimes_{k} \mathcal{O} \stackrel{\alpha_{2}}{\longrightarrow} F \otimes \Sigma_{2}$. There is an induced map $W \otimes \Sigma_{2}^{-2} \stackrel{\alpha_{2}}{\longrightarrow} F \otimes \Sigma_{1}(-1)$ which gives, on the level of $H_{*}^{1}$, the subspace $W(1)$ of $M_{\Sigma_{1}}$ by Lemma 1.2 .

Likewise, we get a map $V \otimes_{k} \mathcal{O} \stackrel{\alpha_{1}}{\longrightarrow} F \otimes \Sigma_{1}$. Together, they give a map

$$
\left(V \otimes \Sigma_{1}^{-1}\right) \oplus\left(W \otimes \Sigma_{2}^{-1}\right) \stackrel{\left(\alpha_{1}, \alpha_{2}\right)}{\longrightarrow} F .
$$

To find a vector bundle as the quotient, we will add an $L^{\prime}$ to $F$. Specifically, consider the dual map $F^{\vee} \stackrel{\left[\alpha_{1}, \alpha_{2}\right]^{\vee}}{\longrightarrow}\left[\left(V \otimes \Sigma_{1}^{-1}\right) \oplus\left(W \otimes \Sigma_{2}^{-1}\right)\right]^{\vee}$. We can find a free $S(\mathcal{Q})$-module surjecting onto the graded module $C=\operatorname{coker} H_{*}^{0}\left([\alpha, \beta]^{\vee}\right)$ and then lift the surjection to get a map to $H_{*}^{0}\left(\left[\left(V \otimes \Sigma_{1}^{-1}\right) \oplus\left(W \otimes \Sigma_{2}^{-1}\right)\right]^{\vee}\right)$. If $L^{\prime \vee}$ is the free bundle obtained by sheafifying this free module, we get a surjection of vector bundles

$$
F^{\vee} \oplus L^{\prime \vee} \stackrel{\left[\begin{array}{ll}
\alpha_{1}^{\vee} & * \\
\alpha_{2}^{\vee} & *
\end{array}\right]}{\longrightarrow}\left[\left(V \otimes \Sigma_{1}^{-1}\right) \oplus\left(W \otimes \Sigma_{2}^{-1}\right)\right]^{\vee}
$$

which is also a surjection on the level of all global sections.

Define $E$ as the dual of the kernel of this map. We get the sequence

$$
0 \rightarrow\left(V \otimes \Sigma_{1}^{-1}\right) \oplus\left(W \otimes \Sigma_{2}^{-1}\right) \stackrel{\left[\begin{array}{cc}
\alpha_{1} & \alpha_{2} \\
* & *
\end{array}\right]}{\longrightarrow} F \oplus L^{\prime} \rightarrow E \rightarrow 0 .
$$


By our construction and Serre duality, the map $\left[\begin{array}{cc}\alpha_{1} & \alpha_{2} \\ * & *\end{array}\right]$ induces an inclusion on the level $H^{2}$, hence $H_{*}^{1}(F) \cong H_{*}^{1}(E)$.

Next, consider the sequence $0 \rightarrow F \oplus L^{\prime} \stackrel{\left[\begin{array}{ll}* & 0 \\ 0 & I\end{array}\right]}{\longrightarrow} L_{1} \oplus L^{\prime} \stackrel{[\phi, 0]}{\longrightarrow} L_{0} \rightarrow 0$. We get the composite inclusion $\left(V \otimes \Sigma_{1}^{-1}\right) \oplus\left(W \otimes \Sigma_{2}^{-1}\right) \rightarrow F \oplus L^{\prime} \rightarrow L_{1} \oplus L^{\prime}$. This inclusion has a dual $\operatorname{map}\left[L_{1} \oplus L^{\prime}\right]^{\vee} \rightarrow\left[\left(V \otimes \Sigma_{1}^{-1}\right) \oplus\left(W \otimes \Sigma_{2}^{-1}\right)\right]^{\vee}$ which is surjective on global sections. Since a minimal surjection to $\Sigma_{i}$ is given in the canonical sequences (11), it follows that the kernel can be identified with $\left(V^{\vee} \otimes \Sigma_{1}^{-1}\right) \oplus\left(W^{\vee} \otimes \Sigma_{2}^{-1}\right) \oplus P^{\vee}$, where $P$ is a free summand of $L_{1} \oplus L^{\prime}$. This results in the commuting diagram

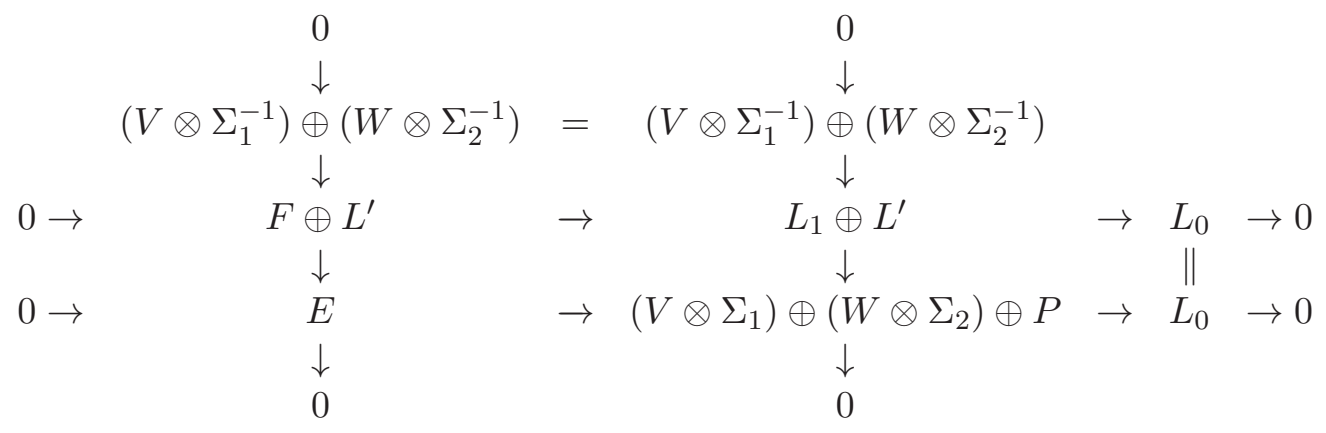

The lowest row can be compared with the sequence $0 \rightarrow E \rightarrow A_{E} \rightarrow L_{0} \rightarrow 0$. In both sequences, $H_{*}^{0}\left(L_{0}\right)$ is mapped surjectively and minimally to $H_{*}^{1}(E)$, hence by Lemma 1.5)(2), the two sequences are isomorphic. Therefore $\mu, \nu$ coincide with $\mu_{E}, \nu_{E}$ and the diagram above is a diagram of $E$.

\section{Examples}

\section{Example 4.1.}

Let $M=k$ in degree 0 . Then, denoting $\Omega_{\mathrm{P}^{3}}^{i}$ as $\Omega^{i}$, the vector bundle $F$ associated to $M$ is $F=\Omega_{\mid \mathcal{Q}}^{1}$. For $i=1,2$, the auxiliary modules $M_{\Sigma_{i}}$ are both equal to $k^{\oplus 2}$, concentrated in degree 0 . Hence any element of $M_{\Sigma_{i}}$ is a $\Sigma_{j}$-socle element $(j \neq i)$. There are several choices for $V, W$, each giving a bundle $E$ without ACM summands.

1. Minimally, when $V=W=0, E=F=\Omega_{\mid \mathcal{Q}}^{1}$.

2. In the maximal case, when $V=k^{\oplus 2}$ in degree -1 and $W=k^{\oplus 2}$ in degree $-1(\nu=$ $\nu_{-1}=2$ and $\left.\mu=\mu_{-1}=2\right)$, the bundle $E$ is $\Omega_{\mid \mathcal{Q}}^{2}(2)$. The diagram for $E$ in this case 
requires additional line bundles to supplement $F$ :

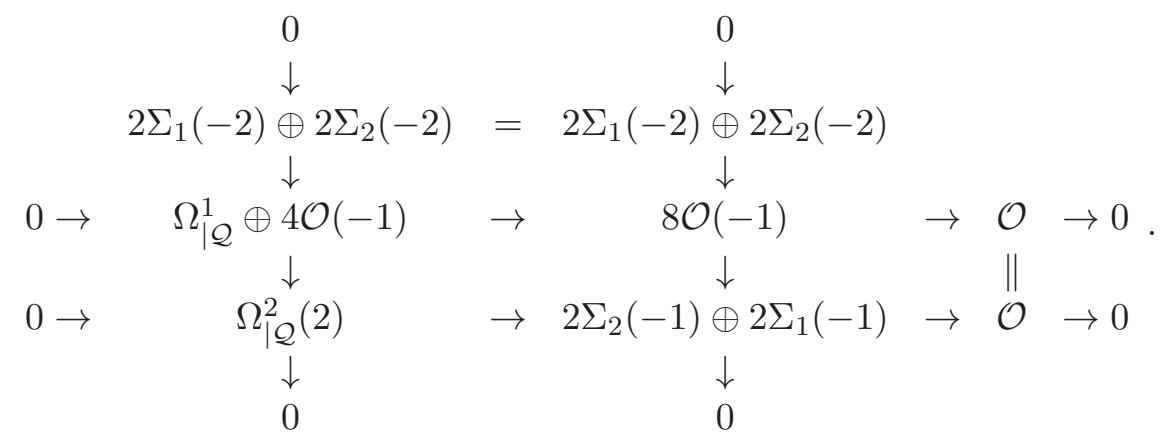

3. When $W=k^{\oplus 2}$ in degree -1 and $V=0\left(\nu=\nu_{-1}=2\right.$ and $\left.\mu=0\right)$, the vector bundle $E$ is $\Sigma_{1}^{-2}=\mathcal{O}(-2,0)$, the kernel of $2 \Sigma_{2}(-1) \rightarrow \mathcal{O}$.

4. In the next three cases, the bundle depends on parameters. When $W=k$ in degree -1 and $V=0\left(\nu=\nu_{-1}=1\right.$ and $\left.\mu=0\right)$, there is a one parameter family of bundles corresponding to the choice of the subspace $W$ of $k^{\oplus 2}$. In fact, $\operatorname{Hom}\left(\Sigma_{1}(-2), \Omega_{\mid \mathcal{Q}}^{1}\right)$ is two dimensional, and each non-zero element gives an inclusion of bundles whose cokernel is a rank two bundle $E$ without ACM summands (giving an $\eta$-sequence). An example of such a bundle $E$ is the rank two bundle given as the kernel of $\Sigma_{2}(-1) \oplus 2 \mathcal{O}(-1) \stackrel{\left[s, x_{2}, x_{3}\right]}{\longrightarrow} \mathcal{O}$ (giving a $\gamma$-sequence).

5. When $W=k^{\oplus 2}$ in degree -1 and $V=k$ in degree $-1\left(\nu=\nu_{-1}=2\right.$ and $\left.\mu=\mu_{-1}=1\right)$, an example of a vector bundle $E$ without ACM summands corresponding to such a choice is the rank two bundle given as the kernel of $2 \Sigma_{2}(-1) \oplus \Sigma_{1}(-1) \stackrel{[s, t, u]}{\longrightarrow} \mathcal{O}$. The bundles in this one-parameter family can be also viewed as extensions of the type $0 \rightarrow \mathcal{O}(-2,0) \rightarrow E \rightarrow \Sigma_{1}(-1) \rightarrow 0$. The diagram is

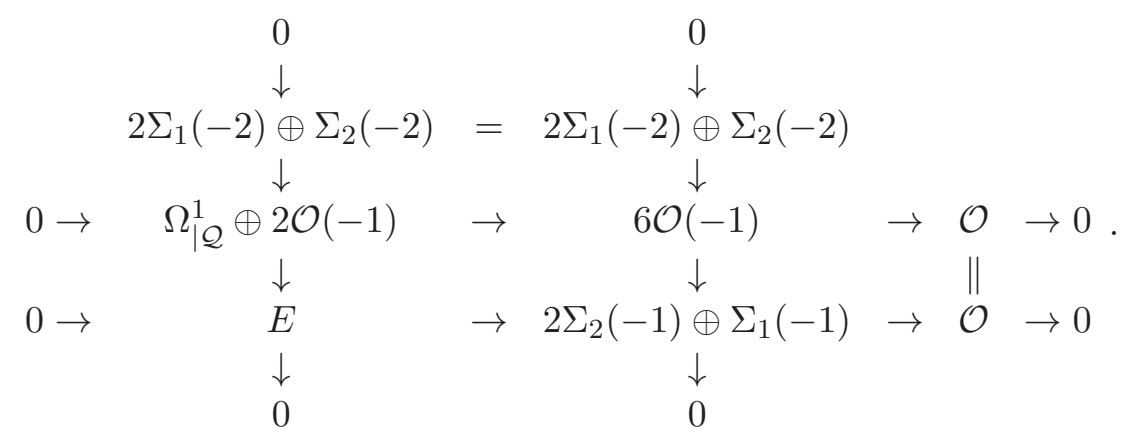

6. When $W=k$ and $V=k$ in degree $-1\left(\nu=\nu_{-1}=1\right.$ and $\left.\mu=\mu_{-1}=1\right)$, the bundles are parametrized by $\mathbf{P}^{1} \times \mathbf{P}^{1}$. Such a bundle $E$ has rank two and has diagram 


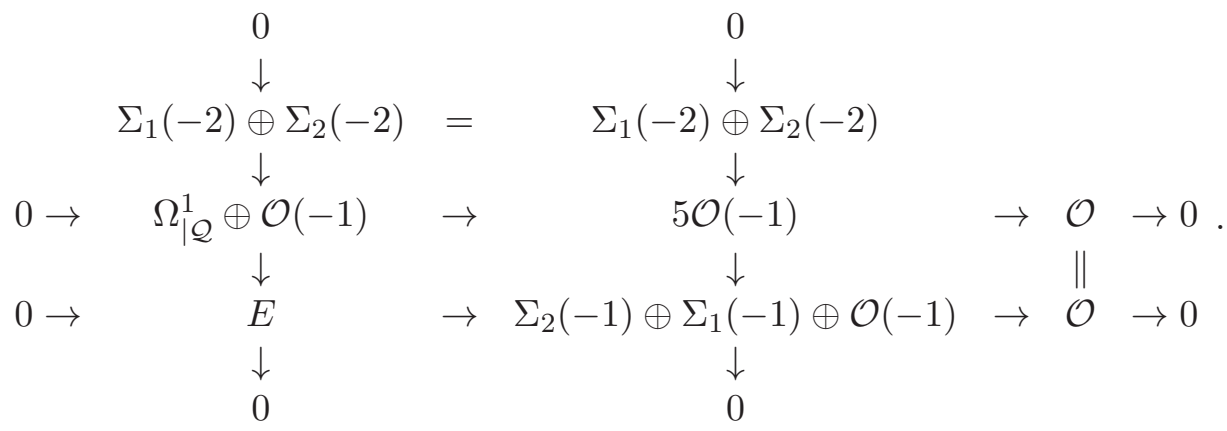

$E(1)$ has a unique section whose zero-scheme is a point on $\mathcal{Q}$.

\section{Example 4.2.}

Let $M=k^{\oplus 2}$ in degree -1 , so $F=2 \Omega_{\mid \mathcal{Q}}^{1}(1)$ and $M_{\Sigma_{i}} \cong k^{\oplus 4}$ in degree -1 for $i=1,2$. Let us consider $V=k^{\oplus 2}$ in degree 0 and $W=k^{\oplus 2}$ in degree $0\left(\nu=\nu_{0}=2\right.$ and $\left.\mu=\mu_{0}=2\right)$. The diagram for a rank two bundle $E$ written below that is associated to these invariants certainly exists for $E$ equal to $[\mathcal{O}(-2,0) \oplus \mathcal{O}(0,-2)](1)$ as the direct sum of diagrams for $\mathcal{O}(-2,0)(1)$ in part $(2)$ of the example above and the corresponding one for $\mathcal{O}(0,-2)(1)$ :

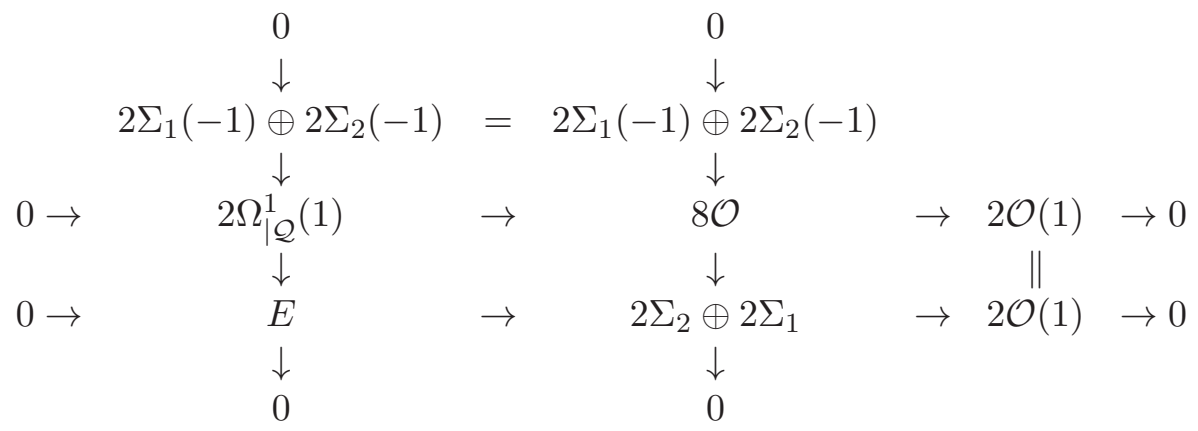

Let $N$ be a null correlation bundle on $\mathbf{P}^{3}$. Then $N$ is a stable bundle of rank 2 on $\mathbf{P}^{3}$ with $c_{1}=0, c_{2}=1$, and is obtained from a sequence

$$
0 \rightarrow \mathcal{O}_{\mathbf{P}^{3}}(-1) \stackrel{s}{\rightarrow} \Omega^{1}(1) \rightarrow N \rightarrow 0 .
$$

Let $E=\left.N\right|_{\mathcal{Q}}$. Then $H^{0}(E)=0, c_{1}=0, c_{2}=2$. Any rank two bundle $E$ on $\mathcal{Q}$ with the properties $H^{0}(E)=0, c_{1}=0, c_{2}=2$ satisfies $M(E)=k^{\oplus 2}$ in degree -1 , using RiemannRoch. Then the $\gamma$-sequence $0 \rightarrow E \rightarrow A_{E} \rightarrow 2 \mathcal{O}(1) \rightarrow 0$ is easily analyzed to show that $A_{E}=2 \Sigma_{2} \oplus 2 \Sigma_{1}$. So $E$ has the indicated diagram.

Any rank two bundle $E$ on $\mathcal{Q}$ with $H^{0}(E)=0, c_{1}=0, c_{2}=2$ is Mumford-Takemoto stable (29]). Le Potier [28] has a stronger definition of stability for such a bundle on $\mathcal{Q}$. Suppose $H^{0}(E)=0, c_{1}=0, c_{2}=2$. If $H^{0}(E(a, b)) \neq 0$ with $b<0$, and if $E(a-1, b)$, $E(a, b-1)$ have no sections, then $a \geq 1$ and a section of $H^{0}(E(a, b))$ determines a subscheme $Z$ with finite support (or empty) with degree $(Z)=2+2 a b \geq 0$. Hence if $H^{0}(E(a, b)) \neq 0$ and either $b<0$ or $a<0$, then $E(a, b)=E(1,-1)$ or $E(-1,1)$. Le Potier stability for a rank two bundle $E$ with $c_{1}=0, c_{2}=2$ requires that $H^{0}(E)=0, H^{0}(E(-1,1))=0, H^{0}(E(1,-1))=0$. This coincides with the notion of a stable projectable bundle due to Soberon-Chavez [38].

Using the invariants $V=k^{\oplus 2} \subset H^{1}\left(2 \Omega_{\mid \mathcal{Q}}^{1}(1) \otimes \Sigma_{2}\right)$ and $W=k^{\oplus 2} \subset H^{1}\left(2 \Omega_{\mid \mathcal{Q}}^{1}(1) \otimes \Sigma_{1}\right)$, we can distinguish three types of bundles $E$ on $\mathcal{Q}$ with the above diagram: split, non-split non-le 
Potier stable and le Potier stable. For convenience, in $F=2 \Omega_{\mid \mathcal{Q}}^{1}(1)=k^{\oplus 2} \otimes_{k} \Omega_{\mid \mathcal{Q}}^{1}(1)$, let $\left\{e_{1}, e_{2}\right\}$ be a basis for $k^{\oplus 2}$ and let $e_{1} \Omega_{\mid \mathcal{Q}}^{1}(1)$ and $e_{2} \Omega_{\mid \mathcal{Q}}^{1}(1)$ denote the corresponding summands.

1. We have already seen that if $W=H^{1}\left(e_{1} \Omega_{\mid \mathcal{Q}}^{1}(1) \otimes \Sigma_{1}\right)$ and $V=H^{1}\left(e_{2} \Omega_{\mid \mathcal{Q}}^{1}(1) \otimes \Sigma_{2}\right)$, then $E$ is split as $[\mathcal{O}(-2,0) \oplus \mathcal{O}(0,2)](1)$.

2. If $W=H^{1}\left(e_{1} \Omega_{\mid \mathcal{Q}}^{1}(1) \otimes \Sigma_{1}\right)$ (without conditions on $V$ ), we get an inclusion of left columns of the diagrams for $\mathcal{O}(-2,0)(1)$ and $E$, yielding a short sequence

$$
0 \rightarrow \mathcal{O}(-1,1) \rightarrow E \rightarrow \mathcal{O}(1,-1) \rightarrow 0 .
$$

$E$ is not le Potier stable since $H^{0}(E(1,-1)) \neq 0$.

Conversely, if $E$ is not le Potier stable, and also satisfies our diagram, it must be that $h^{0}(E(1,-1)) \neq 0$ or $h^{0}(E(-1,1)) \neq 0$. By a Chern class computation, we get, in the first case, an exact sequence $0 \rightarrow \mathcal{O}(-1,1) \rightarrow E \rightarrow \mathcal{O}(1,-1) \rightarrow 0$. The left columns of the diagrams for $\mathcal{O}(-2,0)(1)$ and $E$ can be completed to a commuting diagram since $\operatorname{Ext}^{1}\left(\Omega_{\mid \mathcal{Q}}^{1}(1), \Sigma_{i}(-1)\right)=0$. It follows that for some basis $\left\{e_{1}, e_{2}\right\}, E$ is obtained with $W=H^{1}\left(e_{1} \Omega_{\mid \mathcal{Q}}^{1}(1) \otimes \Sigma_{1}\right)$.

3. For general choices of $V, W$, the $E$ we obtain is a le Potier stable bundle on $\mathcal{Q}$, giving a point in the moduli space $M_{\mathcal{Q}}^{l p}(0,2)$ of le Potier stable bundles.

Le Potier 28 analyzes the restriction to the quadric $\mathcal{Q}$ of null correlation bundles $N$. Let $M_{\mathbf{P}^{3}}^{0}(0,1)$ be the open subset of $M_{\mathbf{P}^{3}}(0,1)$ consisting of all bundles $N$ such that $\left.N\right|_{\mathcal{Q}}$ is le Potier stable on $\mathcal{Q}$. He shows that restriction gives an ètale quasi-finite morphism from $M_{\mathbf{P}^{3}}^{0}(0,1)$ onto an open proper subset $U \subset M_{\mathcal{Q}}^{l p}(0,2)$. The generic bundle $E$ of $U$ has a twin pair (a Tjurin pair) of null correlation bundles restricting to it, while there are bundles $E$ in $U$ with a unique null correlation bundle restricting to it. Soberon-Chavez [38] compactifies $M_{\mathcal{Q}}^{l p}(0,2)$ using only the non-split non-le Potier stable bundles described above.

A complete discussion of the diagram in this example, which we have not carried out, would describe how the choices of $V, W$ determine whether the bundle $E$ obtained is in the subset $U$ of $M_{\mathcal{Q}}^{l p}(0,2)$, and if so, if $E$ has a Tjurin pair over it. We have also not discussed strictly semi-stable bundles with $c_{1}=0, c_{2}=2$ on $\mathcal{Q}$ with $H^{0}(E) \neq 0$ since such bundles $E$ have a different $M(E)$, hence a different diagram. However, we give an example below of a bundle not in $U$.

If $E$ is a bundle with the diagram above, then the $\gamma$-sequence gives rise to a homomorphism $g=\left(g_{1}, g_{2}\right): 2 \Sigma_{2} \oplus 2 \Sigma_{1} \rightarrow 2 \mathcal{O}(1)$ and $E$ is le Potier stable if and only if both det $g_{1}$, det $g_{2}$ (quadratics in $s, t$, respectively $u, v$ ) are non-zero. It is easy to calculate that for such an $E$, the jumping lines contained in the two pencils on $\mathcal{Q}$ are obtained from the zeroes of $\operatorname{det} g_{1}$ and $\operatorname{det} g_{2}$. The restriction of a null-correlation bundle $N$ to $\mathcal{Q}$ has a $\gamma$-sequence which can be calculated. When $\left.N\right|_{\mathcal{Q}}$ is le Potier stable, we can verify le Potier's observation about the jumping lines of $\left.N\right|_{\mathcal{Q}}$ and see that it is not possible for both $\operatorname{det} g_{1}$ and $\operatorname{det} g_{2}$ to have repeated roots. Now consider the map

$$
g=\left[\begin{array}{cccc}
s & t & u & v \\
-t & s-2 t & v & 0
\end{array}\right]
$$

We can check that $g$ is a surjection of bundles on $\mathcal{Q}$, giving a rank two bundle $E$, and that $H^{0}(E)=H^{0}(E(1,-1))=H^{0}(E(-1,1))=0$. Hence this $E$ is le Potier stable but not in the image $U$ of restrictions of null-correlation bundles. 


\section{References}

[1] E. Arrondo, L. Costa, Vector bundles on a Fano threefolds without intermediate cohomology, Comm. Algebra 28, No. 8, 3899-3911 (2000).

[2] E. Arrondo, D. Faenzi, Vector bundles with no intermediate cohomology on Fano threefolds of type $V_{22}$, Pacific J. Math. 225, No. 2, 201-220 (2006).

[3] E. Arrondo, B. GrañA, Vector bundles on $G(1,4)$ without intermediate cohomology, J. of Algebra 214, 128-142 (1999).

[4] E. Arrondo, F. Malaspina, Cohomological Characterization of Vector Bundles on Grassmannians of Lines, J. of Algebra 323, 1098-1106 (2010).

[5] P. BAKhtaRy, Splitting criteria for vector bundles on higher-dimensional varieties, Pacific J of Math. Vol. 252, No 1, 19-30 (2011).

[6] E. Ballico, F. Malaspina, Qregularity and an Extension of the Evans-Griffiths Criterion to Vector Bundles on Quadrics, J. Pure Appl. Algebra 213, n. 2, 194-202 (2009).

[7] E. Ballico, F. Malaspina, Regularity and Cohomological Splitting Conditions for Vector Bundles on Multiprojectives Spaces, J. of Algebra 345, 137-149 (2011).

[8] W. Barth, K. Hulek, Monads and moduli of vector bundles, Manuscripta Math. 25, 323-447 (1978).

[9] J.Biswas , G. V. Ravindra, Arithmetically Cohen-Macaulay bundles on complete intersection varieties of sufficiently high multi-degree, Math. Z. 265, No. 3, 493-509 (2010).

[10] C. Brambilla, D. Faenzi, Moduli space of rank 2 ACM bundles on prime Fano threefolds, Michigan Math. J. 60 No. 1, 113-148 (2011).

[11] R.-O. Buchweitz, G.-M. Greuel And F.-O. Schreyer, Cohen-Macaulay modules on hypersurface singularities II, Invent. Math. 88, 165-182 (1987).

[12] A. Buraggina, The intermediate cohomology modules of a rank two vector bundle on $\mathbf{P}^{3}$ are not split, Comm. Algebra 25, No. 3, 999-1008 (1997).

[13] M. Casanellas, R. Hartshorne, ACM bundles on cubic surfaces, J. Eur. Math. Soc. Vol. 13, Issue 3, 709-731 (2011).

[14] M. Casanellas, R. Hartshorne, F. Geiss, F-O Schreyer, Stable Ulrich Bundles, Int. J. of Math. Vol 23 No 08, 1250083 (2012).

[15] L. Chiantini, D. Faenzi, Rank 2 arithmetically Cohen-Macaulay bundles on a general quintic surface, Math. Nachr. 282 No. 12, 1691-1708 (2009).

[16] L. Chinntini, C. Madonna, ACM bundles on a general quintic threefold, Dedicated to Silvio Greco on the occasion of his 60th birthday (Catania 2001). Matematiche 55, no. 2, 239-258 (2000).

[17] L. Chiantini, C. Madonna, A splitting criterion for rank 2 bundles on a general sextic threefold, Internat. J. Math. 15, No. 4, 341-359 (2004). 
[18] L. Chiantini, C. Madonna, ACM bundles on general hypersurfaces in $\mathbf{P}^{5}$ of low degree, Collect. Math. Vol. 56 No.1, 85-96 (2005).

[19] L. Chiantini, P. Valabrega, Subcanonical curves and complete intesections in the projective 3-space, Ann. Mat. Pura Appl. 138, 309-330 (1984).

[20] L. Costa And R. M. Miró-Roig, Cohomological characterization of vector bundles on multiprojective spaces, J. Algebra 294, No. 1, 73-96 (2005), with a corrigendum in 319, No. 3, 1336-1338 (2008).

[21] L. Costa, R. M. Miró-Roig, Homogeneous Ulrich bundles on Grassmannians of lines, Preprint.

[22] L. Ein An analogue of Max Noether's theorem, Duke Math. J. 52, No.3, 689-706 (1985).

[23] E.G. Evans, P. Griffiths, The syzygy problem, Ann. of Math. 114, No. 2, 323-333 (1981).

[24] D. Faenzi, Bundles over the Fano threefold V ${ }_{5}$, Comm. Algebra, 33(9), 3061-3080 (2005).

[25] D. FAEnZI, Rank 2 arithmetically Cohen-Macaulay bundles on a nonsingular cubic surface, J. Algebra 319, 143-186 (2008).

[26] G. Horrocks, Vector bundles on the punctured spectrum of a ring, Proc. London Math. Soc. (3) 14, 689-713 (1964).

[27] H. KNÖRRER, Cohen-Macaulay modules of hypersurface singularities I, Invent. Math. 88, 153-164 (1987).

[28] J. Le Potien, Sur l'espace de modules des fibrés de Yang et Mills, Mathematics and physics (Paris, 1979/1982), Progr. Math. 37, 65-137, Birkhäuser Boston (1983).

[29] M. Maruyama, Stable vector bundles on an algebraic surface, Nagoya Math. J. 58, 25-68 (1975).

[30] N.Mohan Kumar, C. Peterson, A.P. RaO, Monads on projective spaces, Manuscripta Math. 112, 183-189 (2003).

[31] N.Mohan Kumar, A. P. Rao, G. V. Ravindra, Arithmetically Cohen-Macaulay bundles on hypersurfaces, Comment. Math. Helv. 82, No. 4, 829-843 (2007).

[32] N.Mohan Kumar, A. P. Rao, G. V. Ravindra, Arithmetically Cohen-Macaulay bundles on three dimensional hypersurfaces, Int. Math. Res. Not. IMRN, no. 8, Art. ID rnm025, 11 pp (2007).

[33] N.Mohan Kumar, A. P. Rao, G. V. Ravindra, On Codimension Two Subvarieties In Hypersurfaces, Motives and algebraic cycles, 167-174, Fields Inst. Commun., 56, Amer. Math. Soc., Providence, RI, (2009).

[34] G. Ottaviani, Spinor bundles on quadrics, Trans. Am. Math. Soc. 307, No 1, 301-316 (1988).

[35] G. Ottaviani, Some extensions of Horrocks criterion to vector bundles on Grassmannians and quadrics, Annali Mat. Pura Appl. (IV) 155, 317-341 (1989). 
[36] A. P. RAO, A note on cohomology modules of rank two bundles, J. Algebra 86, 23-34 (1984).

[37] G. V. RAvindra, Curves on threefolds and a conjecture of Griffiths-Harris, Math. Ann. 345, No. 3, 731-748. (2009).

[38] S. Soberon-Chavez, Rank 2 vector bundles over a complex quadric surface, Quart. J. Math. Oxford Ser. (2) 36, 159-172 (1985). 